ARTICLE

\title{
Large arteriolar component of oxygen delivery implies a safe margin of oxygen supply to cerebral tissue
}

Sava Sakadžić1, Emiri T. Mandeville², Louis Gagnon¹,3, Joseph J. Musacchia1, Mohammad A. Yaseen', Meryem A. Yucel ${ }^{1}$, Joel Lefebvre ${ }^{3}$, Frédéric Lesage ${ }^{3}$, Anders M. Dale ${ }^{4}$, Katharina Eikermann-Haerter ${ }^{5}$, Cenk Ayata ${ }^{5,6}$, Vivek J. Srinivasan ${ }^{1, \dagger}$, Eng H. Lo ${ }^{2}$, Anna Devor ${ }^{1,4} \&$ David A. Boas ${ }^{1}$

What is the organization of cerebral microvascular oxygenation and morphology that allows adequate tissue oxygenation at different activity levels? We address this question in the mouse cerebral cortex using microscopic imaging of intravascular $\mathrm{O}_{2}$ partial pressure and blood flow combined with numerical modelling. Here we show that parenchymal arterioles are responsible for $50 \%$ of the extracted $\mathrm{O}_{2}$ at baseline activity, and the majority of the remaining $\mathrm{O}_{2}$ exchange takes place within the first few capillary branches. Most capillaries release little $\mathrm{O}_{2}$ at baseline acting as an $\mathrm{O}_{2}$ reserve that is recruited during increased neuronal activity or decreased blood flow. Our results challenge the common perception that capillaries are the major site of $\mathrm{O}_{2}$ delivery to cerebral tissue. The understanding of oxygenation distribution along arterio-capillary paths may have profound implications for the interpretation of blood-oxygen-level dependent (BOLD) contrast in functional magnetic resonance imaging and for evaluating microvascular $\mathrm{O}_{2}$ delivery capacity to support cerebral tissue in disease.

\footnotetext{
${ }^{1}$ Optics Division, MHG/MIT/HMS Athinoula A Martinos Center for Biomedical Imaging, Department of Radiology, Massachusetts General Hospital and Harvard Medical School, Charlestown, Massachusetts 02129, USA. ${ }^{2}$ Neuroprotection Research Laboratory, Departments of Radiology and Neurology, Massachusetts General Hospital and Harvard Medical School, Charlestown, Massachusetts 02129, USA. ${ }^{3}$ Département de génie électrique, École Polytechnique de Montréal, Montréal, Québec, Canada H3C3A7. ${ }^{4}$ Departments of Radiology and Neurosciences, University of California San Diego, La Jolla, San Diego, California 92093, USA. ${ }^{5}$ Neurovascular Research Laboratory, Department of Radiology, Massachusetts General Hospital and Harvard Medical School, Charlestown, Massachusetts 02129, USA. ${ }^{6}$ Stroke Service and Neuroscience Intensive Care Unit, Department of Neurology, Massachusetts General Hospital and Harvard Medical School, Boston, Massachusetts 02114, USA. †Present address: Department of Biomedical Engineering, University of California Davis, Davis, California 95616, USA. Correspondence and requests for materials should be addressed to S.S. (email: sava.sakadzic@mgh.harvard.edu).
} 
A unique and highly specialized vascular network supports the metabolic needs of the cerebral cortex-a computationally advanced and energetically demanding part of the brain responsible for our higher cognitive functions. Since the cortex relies almost exclusively on oxidative metabolism of glucose $^{1}$, an uninterrupted supply of oxygen to the brain tissue is likely one of the key requirements that defines the structural organization of the cerebrovascular network and blood flow control. The global architecture of the blood supply to the cortex consists of a planar mesh of pial arteries and veins that dive into the cortex supplying the complex microvascular network and draining the blood back to the surface. However, in spite of extensive efforts in brain ${ }^{2-4}$ and in other organs ${ }^{5-10}$, the detailed intravascular oxygen distribution along the microvascular paths that connect pial arteries and veins remains largely unknown ${ }^{11}$. Therefore, we have limited knowledge about the mechanisms that secure sufficient oxygen delivery in microvascular domains during brain activation, and provide some metabolic reserve capacity in diseases that affect either microvascular networks or the regulation of cerebral blood flow (CBF). Such information is therefore critical for our understanding of not only normal brain physiology, but also the relation between progression of microvascular dysfunction and neurodegeneration in various brain diseases ${ }^{12}$, and for attempts to develop a quantitative interpretation of existing and emerging brain-imaging modalities $^{13-15}$.

Until recently the limited knowledge about cortical microvascular oxygen distribution was largely due to a lack of imaging tools for high-resolution deep imaging of cortical oxygenation. To address this challenge, we developed and applied a multimodal microscopy imaging set-up based on 'two-photon $\mathrm{PO}_{2}$ microscopy' - a recently developed technology that can provide maps of oxygen partial pressure $\left(\mathrm{PO}_{2}\right)$ with sub-capillary resolution in cortical arterioles, capillaries, venules and tissue ${ }^{16-19}$. We used two-photon microscopy (TPM) to measure $\mathrm{PO}_{2}$ in a large subset of arterioles, venules and capillaries at different levels of $\mathrm{CBF}$, and to obtain microvascular morphology. We also incorporated in the multimodal imaging set-up a Doppler optical coherence tomography (OCT) imaging set-up ${ }^{20}$, which was exploited to acquire $\mathrm{CBF}$ in penetrating arterioles and surfacing venules to confirm either induced changes in CBF or maintenance of stable $\mathrm{CBF}$ during $\mathrm{PO}_{2}$ measurements. The measurements were combined with a detailed analysis of the microvascular morphology and with computation of oxygen delivery from an anatomical vascular model under different levels of oxygen metabolism to address two basic questions related to cerebral microvascular oxygenation distribution under baseline conditions and during blood flow and metabolic perturbations: we asked 'how much oxygen is extracted from cortical arterioles?' to examine the conventional notion that capillaries are the dominant sites of oxygen delivery to brain tissue under baseline conditions. We also asked 'how is oxygen distributed along the arteriolar and capillary paths at different levels of CBF and tissue oxygen metabolism?' The results of these enquiries reveal the way in which the three-dimensional (3D) cortical microcirculation ensures tissue oxygenation during baseline conditions, as well as the dynamic shift of oxygen extraction along the arteriocapillary path that ensures a safe margin of cerebral tissue oxygenation during metabolic and blood flow perturbations.

We have found that arterioles are responsible for $50 \%$ of the extracted $\mathrm{O}_{2}$ at baseline activity. Most of the remaining $\mathrm{O}_{2}$ exchange is taking place at the level of the first few capillary branches after precapillary arterioles, while majority of the capillaries (those of higher branching orders) on average release little $\mathrm{O}_{2}$ at rest. Our measurements and modelling results support this finding showing that high-branching-order capillaries may act as a dynamic $\mathrm{O}_{2}$ reserve that is recruited on demand to ensure adequate tissue oxygenation during increased neuronal activity or decreased blood flow. Our results challenge the common perception that $\mathrm{O}_{2}$ is almost exclusively released from the capillaries and provide a novel understanding of the distribution and dynamics of $\mathrm{O}_{2}$ extraction along the capillary paths in the cortex.

\section{Results}

Microvascular oxygenation at normal and elevated CBF. We applied two-photon $\mathrm{PO}_{2}$ microscopy to obtain high-resolution $\mathrm{PO}_{2}$ maps in microvascular segments in primary somatosensory (SI) cortex down to a cortical depth of $450 \mu \mathrm{m}$ through a sealed cranial window in mice under isoflurane anaesthesia. Two data sets were obtained representing baseline microvascular oxygenation at normal and elevated CBF (Fig. 1). In the first group of animals $(n=3)$, mice were maintained within the normal range of physiological parameters (that is, normoxic normocapnia). In the second group of animals $(n=3) \mathrm{CBF}$ was globally elevated by

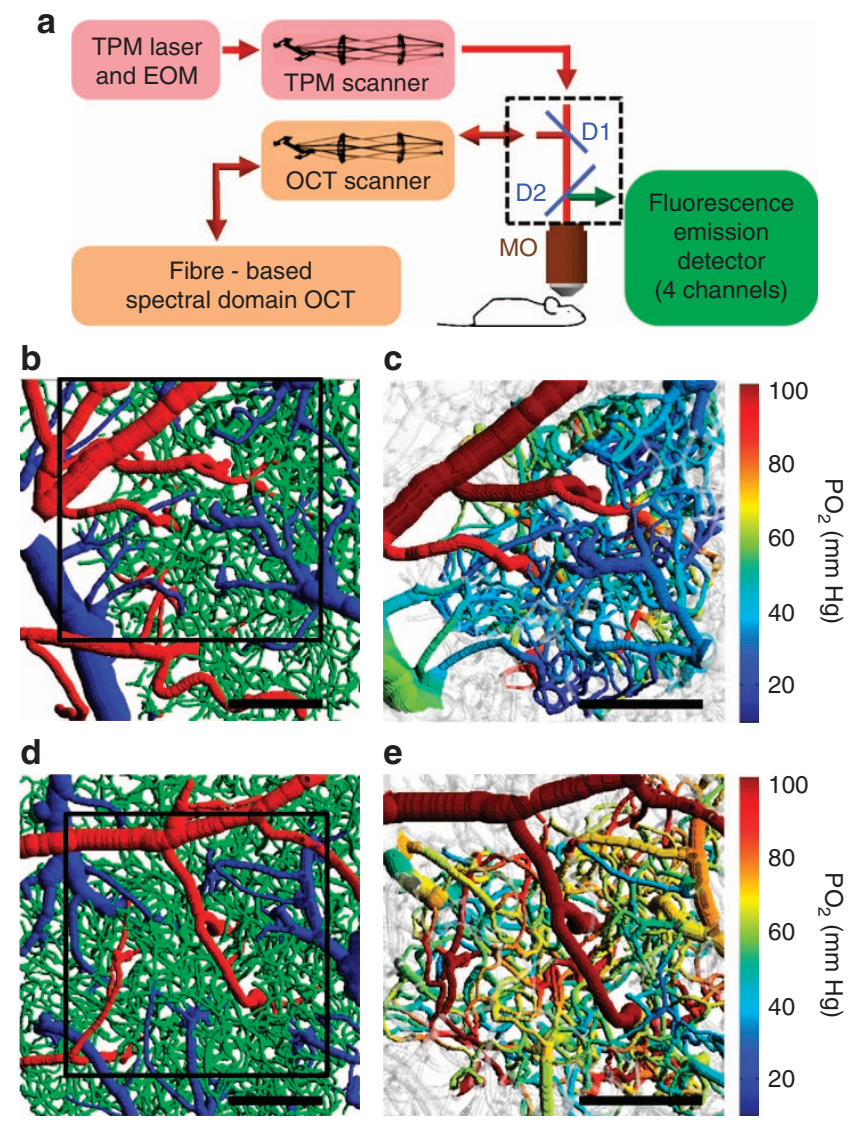

Figure 1 | Imaging set-up and representative images of the microvascular structures and oxygenation. (a) Multimodal microscopy set-up.

Two-photon microscope (TPM) and spectral domain OCT are coupled into the same microscope objective (MO). D1 and D2-dichroic mirrors; EOMelectro-optic modulator; TPM and OCT scanners-galvanometer-mirrorbased scanners together with the scan and tube lenses. (b,d) Top views of the two cortical microvascular stacks with the labelled microvascular segments: arterioles (red), capillaries (green) and venules (blue). (c,e) $\mathrm{PO}_{2}$ measurements during normocapnia (c) and hypercapnia (e) overlaid over microvascular structures presented in $\mathbf{b}$ and $\mathbf{d}$, respectively. Black squares in $\mathbf{b}$ and $\mathbf{d}$ represent $\mathrm{PO}_{2}$ measurement fields of view presented in $\mathbf{c}$ and $\mathbf{e}$, respectively. In grey-coloured vessels $\mathrm{PO}_{2}$ was not estimated. Scale bars, $200 \mu \mathrm{m}$ 
maintaining mild hypercapnia (systemic arterial $\mathrm{PCO}_{2}=45-$ $51 \mathrm{~mm} \mathrm{Hg}$ ) via inhalation of $\sim 5 \% \mathrm{CO}_{2}$. Elevation of the blood flow ( $\sim 30 \%$ increase) during hypercapnia was confirmed using Doppler OCT images of CBF co-registered with the TPM data (Supplementary Fig. 1). Microvascular structure was mapped by TPM at the end of experiments. On the basis of structural images, the microvasculature was segmented, a mathematical graphrepresentation of the microvascular tree was computed and arterioles, venules and capillaries were labelled (Fig. 1b,d). Intravascular $\mathrm{PO}_{2}$ measurements during normocapnia and hypercapnia were subsequently overlaid on the structural microvascular images for further analysis (Fig. 1c,e). As expected, an increase in $\mathrm{CBF}$ in the absence of a significant change in the cerebral metabolic rate of oxygen consumption $\left(\mathrm{CMRO}_{2}\right)$ during hypercapnia led to significant increase in the intravascular $\mathrm{PO}_{2}$ (compare Fig. 1c-e).

Arterioles deliver significant oxygen to the cortical tissue. Our measurements provided two types of evidence that oxygen is readily extracted from the cortical arterioles at baseline conditions: (1) dense cross-sectional $\mathrm{PO}_{2}$ maps inside the diving cortical arterioles during normocapnia revealed pronounced $\mathrm{PO}_{2}$ gradients from the vessel centres to the vessel walls (Fig. 2), indicating oxygen supply to the tissue from the arterioles (for simulation results supporting this observation please see Supplementary Fig. 2). This is consistent with previously documented significant tissue $\mathrm{PO}_{2}$ gradients ${ }^{3,16,18}$ and a marked absence of capillaries in the vicinity of penetrating arterioles ${ }^{21}$. (2) Our measurements along diving arterioles and their branches showed that oxygenation rapidly decreased as blood moved downstream along the arteriolar tree (Fig. 3). $\mathrm{PO}_{2}$ in normocapnia started from above $100 \mathrm{~mm} \mathrm{Hg}\left(\mathrm{SO}_{2} \approx 0.95\right)$ in larger pial arterioles with diameter $\geq 40 \mu \mathrm{m}$ and decayed to $\approx 65 \mathrm{~mm} \mathrm{Hg} \quad\left(\mathrm{SO}_{2} \approx 0.73\right)$ in the smallest arterioles with

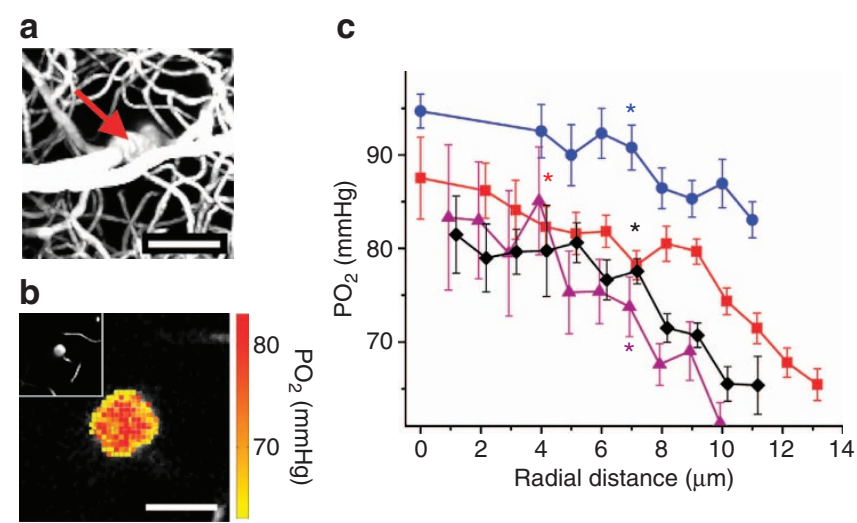

Figure $2 \mid \mathrm{PO}_{2}$ distribution measured inside the penetrating arterioles during normocapnia. (a) Maximum intensity projection of the microvascular structure obtained by TPM showing diving arteriole at the centre (red arrow). Scale bar, $50 \mu \mathrm{m}$. (b) $\mathrm{PO}_{2}$ map inside the penetrating arteriole in $\mathbf{a}, 100 \mu \mathrm{m}$ below the cortical surface. Scale bar, $20 \mu \mathrm{m}$. Inset in the upper left-hand side of $\mathbf{b}$ shows a two-dimensional image from a microvascular stack (a) at the $\mathrm{PO}_{2}$-imaging depth $(100 \mu \mathrm{m}$ below the cortical surface). (c) Radial intra-arteriolar $\mathrm{PO}_{2}$ profiles (radial distance calculated from the vessel axis (vessel centre) to the vessel wall) from four penetrating arterioles similar to the example vessel presented in $\mathbf{a}$ and $\mathbf{b}$. For each $\mathrm{PO}_{2}$ profile, a star indicates the smallest radius where the mean $\mathrm{PO}_{2}$ is significantly lower than the $\mathrm{PO}_{2}$ at the vessel centre (two-sample $t$-test; $P \leq 0.034)$. Data are expressed as mean \pm s.e.m., calculated as detailed in the methods. Results are presented for $n=3$ mice. diameters $<10 \mu \mathrm{m}$, with a decay rate that increased rapidly with decreasing vessel diameter. The mean $\mathrm{PO}_{2}$ from all precapillary arterioles-arteriolar segments immediately proximal to the capillaries-was $66 \mathrm{~mm} \mathrm{Hg} \quad\left(\mathrm{SO}_{2}=0.78\right)$, indicating $\Delta \mathrm{SO}_{2}=0.17$ from the arteriolar segments during normocapnia. This represents $50 \%$ of the $\mathrm{SO}_{2}$ difference between large pial arterioles and venules with diameters $\geq 40 \mu \mathrm{m}\left(\Delta \mathrm{SO}_{2, \mathrm{~A}}\right.$ $\mathrm{v}=0.33$ ). A similar observation can be made by examining the $\mathrm{PO}_{2}$ and $\mathrm{SO}_{2}$ variation with microvascular segment branching order and distance along the microvascular paths with respect to the pial vessels (Supplementary Fig. 3).

An interesting trend of mean $\mathrm{PO}_{2}$ and $\mathrm{SO}_{2}$ increase with increasing venular diameter was observed (Fig. 3), regardless of the exclusion of the pial venules (Supplementary Fig. 4). Further in the Results and in the Discussion sections we provide an explanation for this observation and the impact it has on interpreting results from various imaging modalities.

Finally, during hypercapnia we measured a decrease in oxygen extraction fraction (OEF) from the microvasculature. OEF during hypercapnia was $26 \%\left(\Delta \mathrm{SO}_{2, \mathrm{~A}-\mathrm{V}}=0.25\right)$ in comparison with $\mathrm{OEF}=35 \% \quad\left(\Delta \mathrm{SO}_{2, \mathrm{~A}-\mathrm{V}}=33 \%\right)$ during normocapnia. The decrease in $\mathrm{OEF}$ was paralleled by a measured $\sim 30 \%$ increase in $\mathrm{CBF}$, confirming the expected negligible change in $\mathrm{CMRO}_{2}$. However, under hypercapnia arteriolar oxygen delivery caused only a 0.08 decrease in $\mathrm{SO}_{2}\left(32 \%\right.$ of $\left.\Delta \mathrm{SO}_{2, \mathrm{~A}-\mathrm{V}}\right)$, in comparison with 0.17 decrease in $\mathrm{SO}_{2}\left(50 \%\right.$ of $\left.\Delta \mathrm{SO}_{2, \mathrm{~A}-\mathrm{V}}\right)$ during normocapnia, indicating a significantly increased fractional contribution of capillaries to the oxygen delivery $\left(68 \%\right.$ of $\left.\Delta \mathrm{SO}_{2, \mathrm{~A}-\mathrm{V}}\right)$ during elevated blood flow compared with $\sim 50 \%$ during normocapnia.

Capillary oxygenation adapts to $\mathrm{CBF}$ and $\mathrm{CMRO}_{2}$ perturbations. To address oxygen extraction of capillaries of different branching orders, we analysed the capillary morphology in segmented microvascular networks. This analysis revealed that in the investigated cortical area, the capillary paths between precapillary arterioles and postcapillary venules consisted of, on average, six capillary segments (capillary sections between two consecutive bifurcation points) with a total pathlength of $\sim 340 \mu \mathrm{m}$. There was a good agreement between capillary $\mathrm{PO}_{2}$ measurements at branching orders 2, 3, 4, 5 and 6 going downstream from the precapillary arterioles and capillary $\mathrm{PO}_{2}$ measurements at branching orders 5, 4, 3, 2 and 1 going upstream from the postcapillary venules, respectively (Supplementary Fig. 5). This confirmed that $\mathrm{PO}_{2}$ measurements in the first six capillary segments following precapillary arterioles (Fig. 4) accurately represented the $\mathrm{PO}_{2}$ distribution along the entire capillary path from precapillary arterioles to postcapillary venules.

Two important observations can be made from the mean arteriolar and capillary $\mathrm{PO}_{2}$ and $\mathrm{SO}_{2}$ distribution measurements (Figs 3 and 4): (1) the average capillary segment $\mathrm{PO}_{2}$ and $\mathrm{SO}_{2}$ profile along the capillary path at baseline was strongly nonlinear (Fig. 4c-f). $\mathrm{PO}_{2}$ and $\mathrm{SO}_{2}$ intravascular longitudinal gradients (that is, the rate of $\mathrm{PO}_{2}$ and $\mathrm{SO}_{2}$ decrease along the microvascular segments) in precapillary arterioles and along the first 2-3 capillary segments after precapillary arterioles (that is, lowbranching-order capillaries) were significantly higher than the $\mathrm{PO}_{2}$ and $\mathrm{SO}_{2}$ gradients along capillaries closer to venules (that is, high-branching-order capillaries). This suggests that under baseline conditions, precapillary arterioles and low-branching-order capillaries release on average much more oxygen to the tissue than high-branching-order capillaries; (2) an increase in CBF during hypercapnia demonstrated that while the highly oxygenated arterioles can only modestly increase $\mathrm{SO}_{2}\left(\Delta \mathrm{SO}_{2}=0.11\right.$ in precapillary arterioles), capillaries have the ability to markedly increase $\mathrm{SO}_{2}$ (up to $\Delta \mathrm{SO}_{2}=0.30$ in high-branching-order 

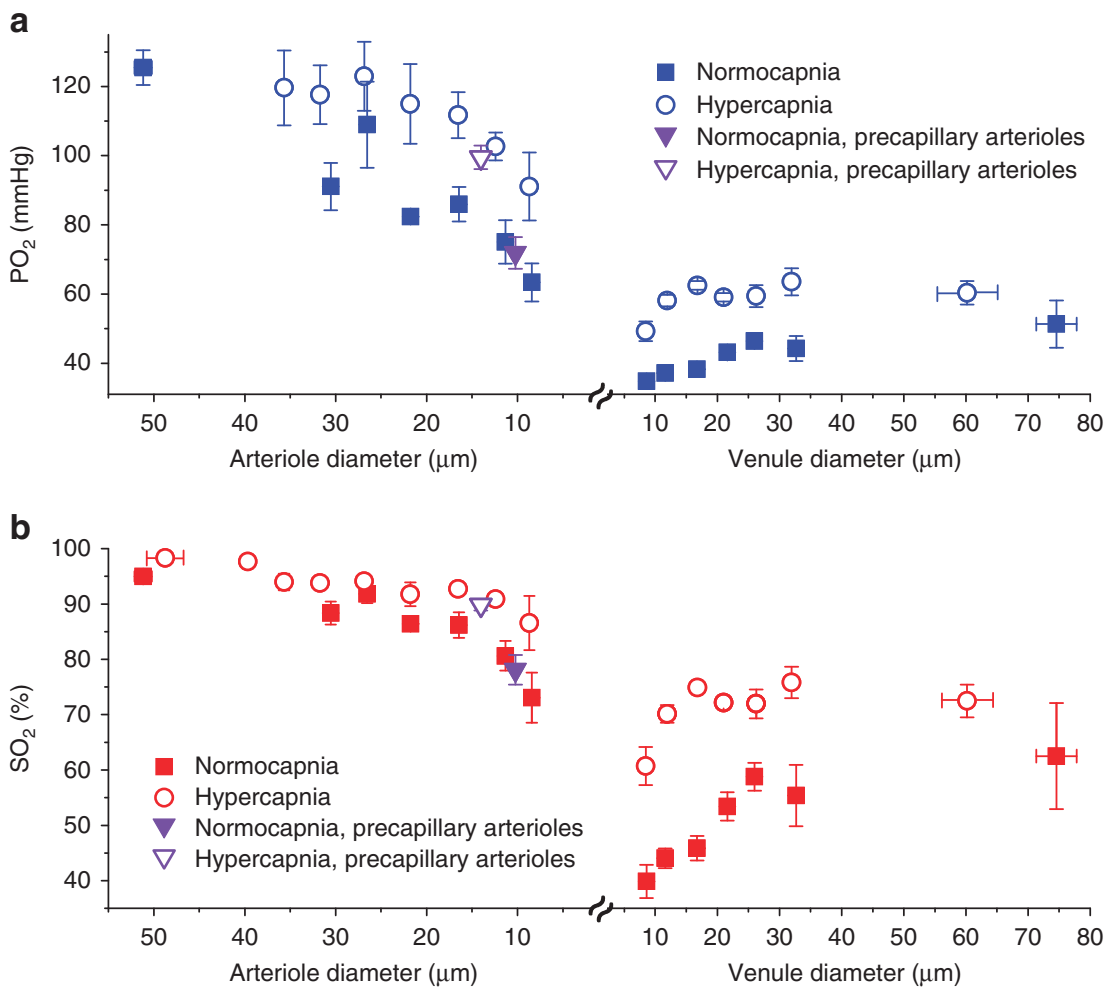

Figure 3 | Oxygen content in arterioles and venules as a function of vessel diameter. (a,b) Intravascular $\mathrm{PO}_{2}$ and $\mathrm{SO}_{2}$, respectively, as a function of arteriolar and venular diameters during normocapnia (solid symbols) and hypercapnia (empty symbols). Inverted triangle symbols represent mean values in precapillary arterioles. All mean $\mathrm{PO}_{2}$ and $\mathrm{SO}_{2}$ during hypercapnia experiments are statistically larger than mean $\mathrm{PO}_{2}$ and $\mathrm{SO}_{2}$ during normocapnia (Student's $t$-test, $P<0.04$, pairwise comparison for diameters $<25 \mu \mathrm{m}$ and between combined measurements for diameters $>25 \mu \mathrm{m}$ ). Data are expressed as mean \pm s.e.m. and were estimated averaging over all vessels over all animals. There were $n=3$ mice for each group.

capillaries) and possibly their contribution to tissue oxygen supply during functional hyperaemia. The following analysis, which is based on these measurements in combination with numerical simulations of oxygen delivery in realistic microvascular anatomical networks, reveals a novel view of cortical oxygen supply in which the cerebral microcirculation keeps tissue oxygen concentration with a safe margin under baseline conditions, as well as during increased metabolic demand or reduced blood flow.

We first asked the question: "what governs oxygen distribution along the capillary path at baseline conditions?" We hypothesized that the nonlinear average $\mathrm{SO}_{2}$ distribution along the capillary paths (Fig. 4) is largely due to greater tissue territories being supplied by low branching order, better oxygenated capillaries. To confirm this hypothesis, we relied on measurements of microvascular morphology and oxygenation, as well as simulations of the blood flow in realistic microvascular networks. We started by considering a simple approximation for the $\mathrm{SO}_{2}$ change along a capillary segment: $\Delta \mathrm{SO}_{2} \approx \mathrm{CMRO}_{2} \times L \times R_{\mathrm{t}}^{2} \times \pi /(\mathrm{CBF} \times 4 \times$ $C_{\mathrm{Hb}}$ ), where $L$ is capillary segment length, $R_{\mathrm{t}}$ is the tissue radius, assuming that each vessel supplies an ideal tissue cylinder, and $C_{\mathrm{Hb}}$ is the haemoglobin concentration in blood. By using this equation and by starting from the $\mathrm{SO}_{2}$ in precapillary arterioles, the $\mathrm{SO}_{2}$ distribution along the capillary branches can be obtained by subsequently subtracting the $\Delta \mathrm{SO}_{2}$ for consecutive capillary branching orders. We assumed that $\mathrm{CMRO}_{2}$ and $C_{\mathrm{Hb}}$ are constant and we used the measured capillary $\mathrm{SO}_{2}$ distribution at rest (Fig. 4e), the measured capillary segment length distribution (Supplementary Fig. 6) and the simulated capillary CBF distribution (Supplementary Fig. 6) and fit the above equation for the tissue radius $R_{\mathrm{t}}$. We estimated an approximately linear $R_{\mathrm{t}}$ decrease from $\sim 60$ to $\sim 10 \mu \mathrm{m}$ for the capillary segments adjacent to precapillary arterioles and postcapillary venules, respectively (Supplementary Fig. 6), consistent with our hypothesis that more-oxygenated low-branching-order capillaries release on average much more oxygen to the tissue than highbranching-order capillaries due to the greater size of their supplied tissue territories. It is interesting to note that the average capillary segment length $L$ changes only slightly from $65.5 \pm 6.2 \mu \mathrm{m}$ for the low-branching-order capillaries to $81.4 \pm 4.4 \mu \mathrm{m} \quad$ (mean \pm s.e.m.) for the high-branching-order capillaries (Supplementary Fig. 6). Our measurements also revealed a remarkably uniform filling of space by the capillaries (Supplementary Figs 6 and 7). If the tissue closest to each capillary segment is deformed into a cylinder, the computed average cylinder radius varies only between $24.2 \pm 2.2$ and $22.3 \pm 1.2 \mu \mathrm{m}$ (mean \pm s.e.m.) for the low-branching-order and high-branching-order capillary segments, respectively. However, our results show that the tissue volumes supplied by the capillaries are not given by the shortest tissue distances to vessels. If they were, $R_{\mathrm{t}}$ would be approximately constant and independent from the capillary branching order. Further, the capillary $\mathrm{SO}_{2}$ distribution would be widely different from the observed $\mathrm{SO}_{2}$ trend (Fig. 4e), as it would be governed by the capillary blood flow distribution and exhibit a concave instead of the measured convex shape of $\mathrm{SO}_{2}$ versus branching order. Instead, the tissue territory supplied by the vessels with higher $\mathrm{PO}_{2}$ is greatly enlarged, overtaking the tissue territory surrounding less-oxygenated vessels. Simple numerical analysis for realistic distances between capillaries (Supplementary Fig. 8) confirms that the tissue territory supplied by the more-oxygenated vessel can grow to include the less-oxygenated vessel. 

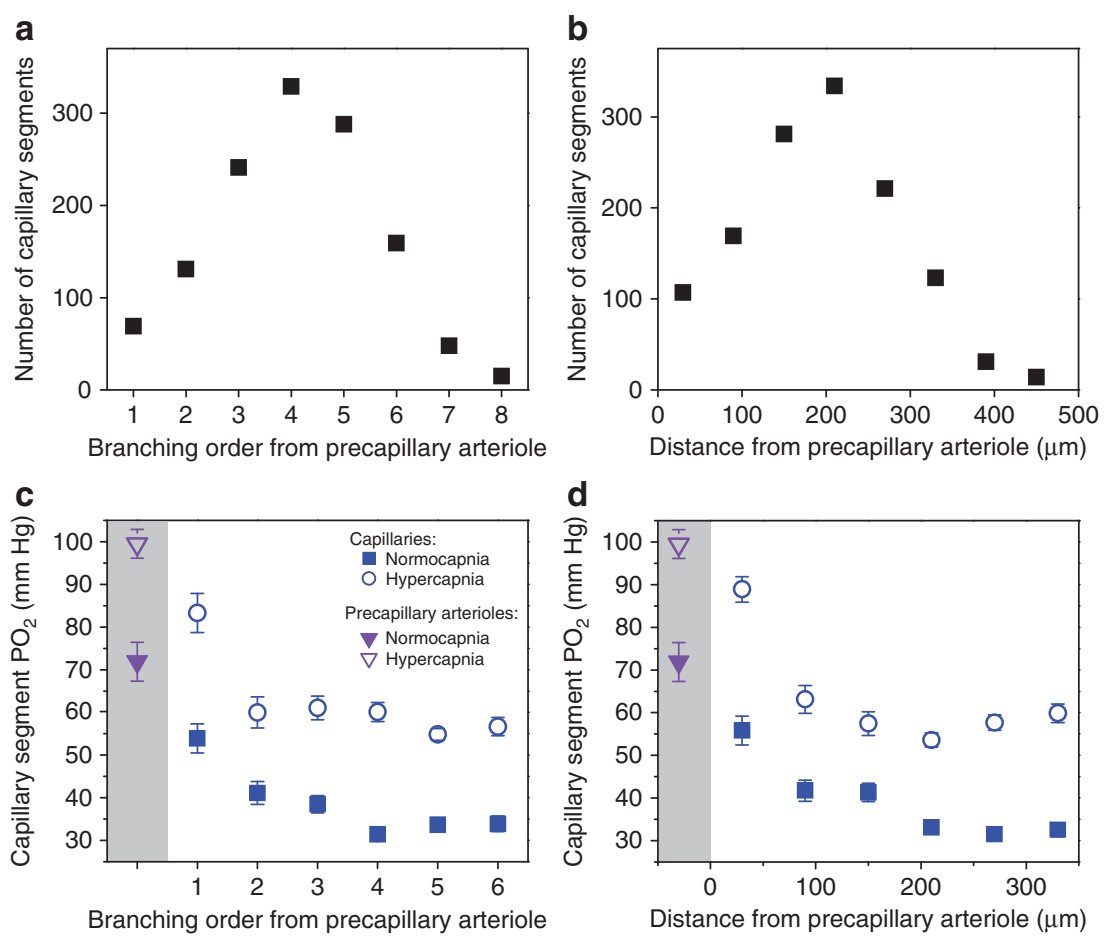

Branching order from precapillary arteriole
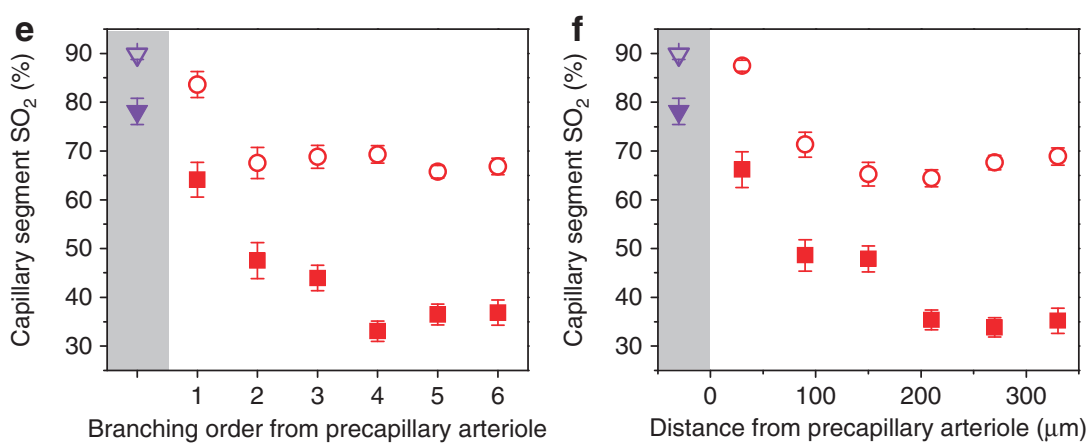

Figure 4 | Capillary oxygen content as a function of branching order and distance from the precapillary arterioles. (a,b) Histograms of the capillary segment number. (c-f) $\mathrm{PO}_{2}$ and $\mathrm{SO}_{2}$ inside the capillary segments. Solid and empty symbols in c-f represent measurements during normocapnia and hypercapnia, respectively. Average $\mathrm{PO}_{2}$ and $\mathrm{SO}_{2}$ values in precapillary arterioles (purple triangle symbols) were plotted inside the shaded regions on the left-hand side of the graphs. At each branching order and distance from precapillary arteriole, mean $\mathrm{PO}_{2}$ and $\mathrm{SO}_{2}$ during hypercapnia experiments are statistically larger than mean $\mathrm{PO}_{2}$ and $\mathrm{SO}_{2}$ during normocapnia (Student's $t$-test, $P<0.006$ ). Data are expressed as mean \pm s.e.m. and were estimated averaging over all vessels over all animals. $n=6$ mice were used for $\mathbf{a}$ and $\mathbf{b}$ and $n=3$ mice were used for each group in $\mathbf{c - f}$.

After elucidating the role of precapillary arterioles and lowand high-branching-order capillaries in baseline tissue oxygen supply, we further asked: 'What governs oxygen distribution along the capillary path during $\mathrm{CBF}$ and $\mathrm{CMRO}_{2}$ perturbations?' In particular, it is critical to consider the responses to decreased $\mathrm{CBF}$ or increased $\mathrm{CMRO}_{2}$-the changes in oxygen supply and consumption encountered during both pathological events and normal brain activation, which have the potential to compromise tissue oxygenation and brain function. Each of these changes, if they happen without significant changes in other physiological parameters, lead to increased $\mathrm{OEF}$ and consequently lower the $\mathrm{PO}_{2}$ and $\mathrm{SO}_{2}$ in the microvascular segments, with the highbranching-order capillaries experiencing the largest decrease in oxygenation due to cumulative $\mathrm{SO}_{2}$ changes along the microvascular paths. Measurements of the oxygenation distribution along the capillary paths (Fig. 4c-f) revealed that lower CBF was associated with both larger $\mathrm{SO}_{2}$ longitudinal gradients in highbranching-order capillaries and their increased fractional contribution to the oxygen release to the tissue. In addition, a simple theoretical analysis shows that even when the oxygen sources (microvascular segments) are kept at constant $\mathrm{PO}_{2}$, an increase in $\mathrm{CMRO}_{2}$ leads to an enlargement of tissue territory supplied by the lower $\mathrm{PO}_{2}$ sources (Supplementary Fig. 8). Our simulations in realistic vascular anatomical networks (VANs) (Supplementary Fig. 9) confirmed that an increase in $\mathrm{CMRO}_{2}$ leads to an increased fractional contribution of downstream capillaries to oxygen delivery and consequently to an increase in their supply territory (Fig. 5). Therefore, in response to the oxygen supply decrease and/or oxygen consumption increase, the less-oxygenated high-branching-order capillaries may experience both an oxygenation decrease and an increase in supplied tissue territory-coordinated changes that may compromise tissue oxygenation. In experiments we observed very high heterogeneity of the capillary oxygenation (Fig. 6), which further emphasizes the importance of keeping the high average $\mathrm{PO}_{2}$ in highbranching-order capillaries at baseline conditions, since the tissue 

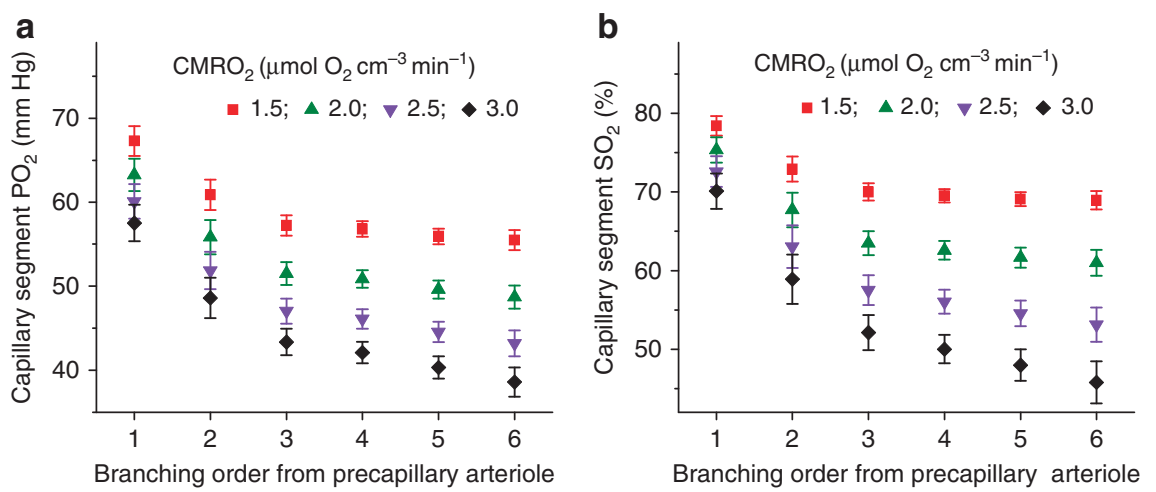

Figure 5 | Influence of $\mathbf{C M R O}_{\mathbf{2}}$ changes on capillary oxygen distribution. Capillary $\mathrm{PO}_{2}$ (a) and $\mathrm{SO}_{2}$ (b) distributions simulated in realistic vascular anatomical network at different levels of $\mathrm{CMRO}_{2}$ and constant $\mathrm{CBF}$. Mean $\mathrm{PO}_{2}$ and $\mathrm{SO}_{2}$ were calculated from the groups of capillaries with different branching order from the precapillary arteriole. Data are expressed as mean \pm s.e.m. and were estimated over all vessels of $n=1 \mathrm{VAN}$.
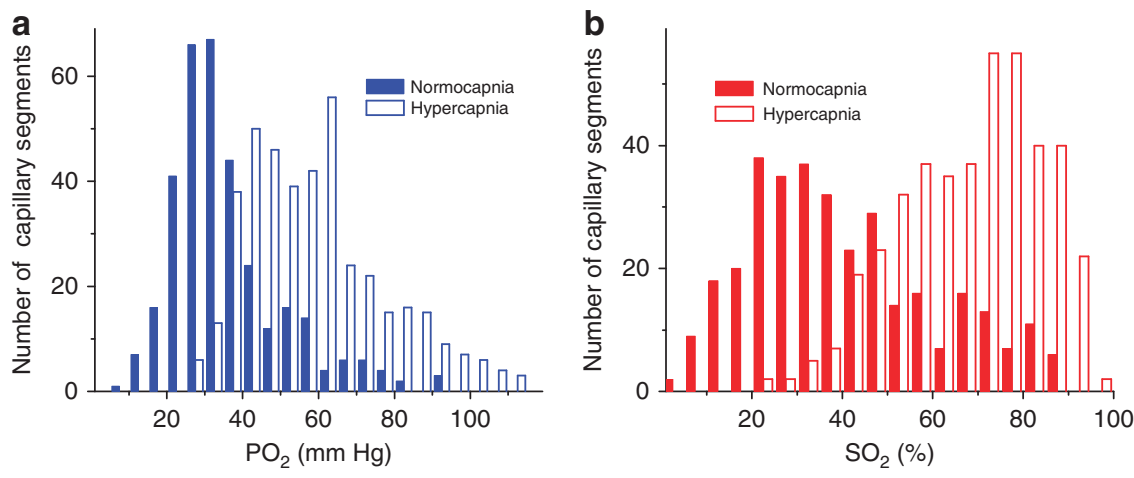

Figure 6 | Capillary $\mathbf{P O}_{\mathbf{2}}$ and $\mathbf{S O}_{\mathbf{2}}$ histograms. Histograms of measured capillary $\mathrm{PO}_{2}(\mathbf{a})$ and $\mathrm{SO}_{2}(\mathbf{b})$ during normocapnia (solid bars) and hypercapnia (empty bars). There were $n=3$ mice for each group.

around capillaries with poorly oxygenated blood may be particularly vulnerable to an OEF increase. In this situation, under baseline conditions, keeping a high $\mathrm{SO}_{2}$ for highbranching-order capillaries and their longitudinal $\mathrm{SO}_{2}$ gradients low (that is, reduced tissue territory supplied by them) may serve as an effective oxygen buffer-a reserve that can be exploited to keep tissue oxygenation in the most vulnerable microvascular domains above a critical level. In addition, we demonstrated the ability of the microvascular network to significantly increase oxygenation in the high-branching-order capillaries during a $\mathrm{CBF}$ increase (Fig. 4). This suggests that during functional hyperaemia-that is, a CBF increase in response to functional activation and elevated metabolism-a further adjustment of oxygen delivery is taking place that results in raising the oxygenation in high-branching-order capillaries to restore the pre-activation state and to preserve a safe margin of tissue oxygenation.

Average $\mathrm{SO}_{2}$ underestimates microvascular oxygen content. Our observation of the venular $\mathrm{PO}_{2}$ increase with cortical vessel diameter (Fig. 3) is similar to observations of other groups in the past in different organs ${ }^{7}$. Several hypotheses have been proposed to explain why mixed venous blood in larger calibre venules has higher oxygenation than capillaries and postcapillary venules: (1) the existence of vascular shunts that will bypass the capillary network and allow direct oxygen advection from arterioles to venules; (2) oxygen diffusion from tissue back to venules, when venules are either close to arterioles or in tissue regions where average $\mathrm{PO}_{2}$ is high; (3) blood oxygenation and flow in a complex microvascular network are highly heterogeneous, and microvascular paths with higher blood flow have higher $\mathrm{PO}_{2}$ and $\mathrm{SO}_{2}$ due to the inverse relation between capillary flow and extraction efficacy. The last hypothesis of a positive correlation between flow and $\mathrm{SO}_{2}$ in microvascular paths implies that the mean $\mathrm{SO}_{2}$ of a population of microvascular segments is lower than the flow-weighted mean $\mathrm{SO}_{2}$ of the same segments, because the segments with high $\mathrm{SO}_{2}$ (and high flow) account for a large fraction of the oxygen transported through all segments. Pial venous $\mathrm{SO}_{2}$, in turn, represents the summed contribution of flowweighted $\mathrm{SO}_{2}$ from many individual microvascular paths, and is therefore always higher than the mean $\mathrm{SO}_{2}$ in the feeding capillaries and postcapillary venules.

Our and other groups' analysis of microvascular morphology 22 does not provide evidence of microvascular shunts in the cortex. On the other hand, oxygen diffusion from the cortical tissue back to venules is taking place, at least sporadically, in the heterogeneously oxygenated cortex ${ }^{19}$. Tissue $\mathrm{PO}_{2}$ may be higher on the cortical surface ${ }^{23}$ due to the influence of large pial arterioles and reduced cortical layer I metabolism. This could cause an increase in pial venular $\mathrm{PO}_{2}$. However, the results from this study confirm a steady $\mathrm{PO}_{2}$ increase in ascending venules below the cortical tissue (Supplementary Fig. 4), away from the influence of pial arterioles. In addition, the ascending venules in primary SI cortex are unlikely targets for sufficient oxygen diffusion from tissue to venules due to their already relatively high flow (that is, short transient time) and reduced ratio of surface to volume. Moreover, our measurements of tissue $\mathrm{PO}_{2}$ in 


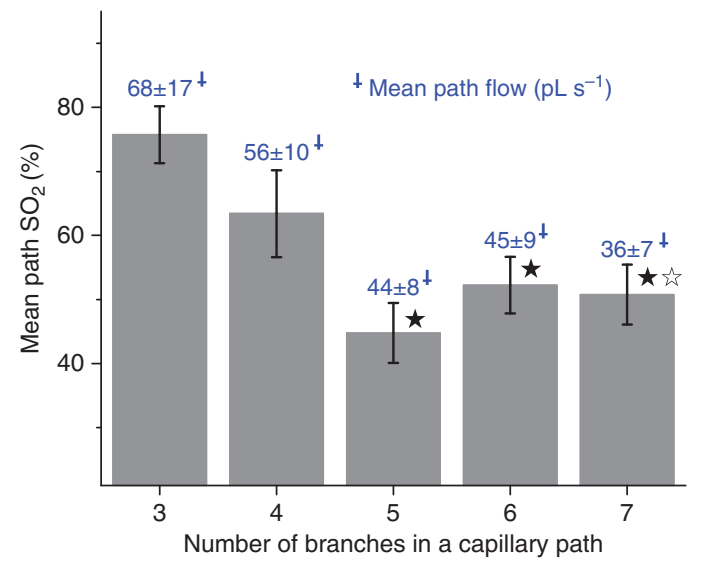

Figure 7 | Correlation between $\mathrm{SO}_{2}$ and blood flow along the capillary paths. Dependence of the mean measured $\mathrm{SO}_{2}$ along the capillary path on a number of branches in a capillary path between precapillary arteriole and postcapillary venule. Simulated blood flow averaged along the same capillary paths is indicated above each bar (blue numbers). Star symbols indicate significant difference (Student's $t$-test, $P<0.05$ ) when comparing with the results for three capillary branches (black asterisk- $\mathrm{SO}_{2}$; white asterisk-blood flow). Data are expressed as mean \pm s.e.m. and were estimated averaging over all vessels over all animals. There were $n=3$ mice.

the forepaw area of primary SI cortex in rats demonstrate small but significant oxygen diffusion from ascending venules to tissue $^{18}$. While these data suggest that oxygen efflux from tissue into the venules is not the major cause of increasing $\mathrm{PO}_{2}$ in the venules, we believe that it is likely that efflux does occur in the high-branching-order capillaries where we have observed flat longitudinal $\mathrm{PO}_{2}$ gradients.

Finally, our results show that the discrepancy between $\mathrm{SO}_{2}$ in pial veins and $\mathrm{SO}_{2}$ in postcapillary venules and downstream capillaries can be largely explained by the difference between a mean $\mathrm{SO}_{2}$ and a flow-weighted mean $\mathrm{SO}_{2}$. On the basis of our measurements during normocapnia, average capillary path $\mathrm{SO}_{2}$ decreases significantly as the number of capillary segments between precapillary arteriole and postcapillary venule increases (Fig. 7). In the same capillary paths, our VAN modelling shows that average capillary path blood flow follows the same trend (Fig. 7), demonstrating that shorter capillary paths indeed have higher $\mathrm{SO}_{2}$ and blood flow on average. Such a characteristic $\mathrm{SO}_{2}$ and flow distribution in the microvasculature results in the mean $\mathrm{SO}_{2}$ in high-branching-order capillaries and postcapillary venules to always be lower than in pial veins. In our VAN modelling, we observed up to a $15 \%$ discrepancy between the mean $\mathrm{SO}_{2}$ and flow-weighted mean $\mathrm{SO}_{2}$ in capillaries (Supplementary Figs $6 \mathrm{~b}$ and 10), suggesting that this effect alone may largely explain why mixed venous blood $\mathrm{SO}_{2}$ is higher than $\mathrm{SO}_{2}$ in postcapillary venules and high-branching-order capillary segments.

\section{Discussion}

We applied a set of novel imaging and analysis tools to assess microvascular oxygenation in mouse primary SI cortex. Until recently, most evidence about cerebral microvascular oxygen distribution was obtained by invasive polarographic microelectrode measurements in the upper several tens of micrometres of the cortex. Our combination of recently developed two-photon $\mathrm{PO}_{2}$ microscopy with TPM imaging of microvascular morphology, Doppler OCT imaging of CBF, microvascular segmentation algorithms and oxygen delivery modelling based on anatomically

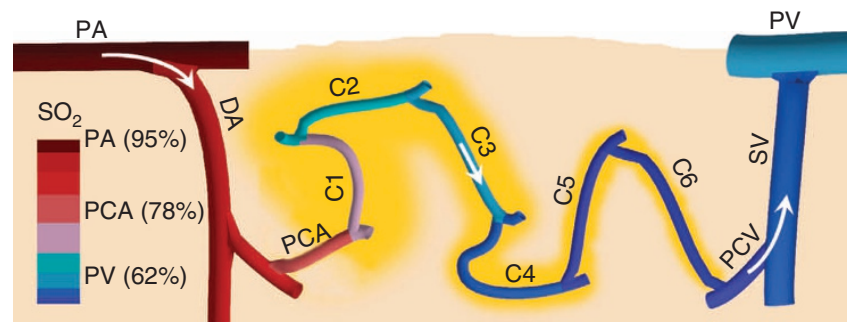

Figure 8 | Simplified representation of the cortical microvascular path, intravascular oxygenation and supplied tissue territories. A vertical crosssection through the cortical tissue revealing one microvascular path close to the cortical surface including pial arteriole (PA), diving arteriole (DA), precapillary arteriole (PCA), capillary path (C1-C6) with the low-branchingorder capillaries (C1-C3) and high-branching-order capillaries (C4-C6), postcapillary venule (PCV), surfacing venule (SV) and pial venule (PV). Arrows represent the direction of the blood flow. Vascular segment colours represent approximate average segment $\mathrm{SO}_{2}$. Tissue territories supplied by capillary segments are outlined in yellow.

accurate microvascular structures, allowed us to obtain very detailed maps of microvascular oxygenation over a substantial depth of the mouse cortex, to quantify the contribution of different microvascular segments to oxygen release to the tissue, and to elucidate shifts in their oxygen delivery during flow and metabolic perturbations.

Our measurements suggest that, contrary to the conventional notion of capillaries being the only sites of oxygen exchange ${ }^{24}$, cortical arterioles represent a significant source of oxygen extraction $(\sim 50 \%)$ to the tissue at baseline conditions. Precapillary arterioles and low-branching-order capillaries release most of the oxygen to the cortical tissue at baseline, while high-branching-order capillaries with their low-resting longitudinal $\mathrm{SO}_{2}$ gradients and reduced supplied tissue territory act as an oxygen delivery buffer, which can be utilized during oxygen consumption increase and blood flow decrease by increasing their delivery contribution to secure an adequate level of tissue oxygenation (Fig. 8).

Cerebral microvascular oxygenation has been investigated for many decades (for a detailed review relevant to this work see Tsai et $\left.a l^{7}\right)$. Arteriolar oxygen extraction in cat and rat cortex ${ }^{2-4}$ as well as in other tissues ${ }^{5}$ were indicated in the past. However, the notion of arteriolar oxygen loss has been questioned due to methodological limitations (for example, limited accuracy of the polarographic electrode oxygen measurements, bias introduced by puncture of vascular walls with the electrode, oxygen consumption by the electrodes and limited number of point measurements near the cortical surface in an open brain preparation), as well as a lack of understanding of the relations between microvascular morphology and the suggested novel view of arteriolar and capillary contribution to oxygen delivery. Some modelling efforts came to the conclusion that significant arteriolar oxygen delivery is present in the skeletal muscles ${ }^{25}$, but without support from the detailed measurements of the microvascular oxygenation and without using the exact microvascular morphology. We believe that our extensive measurement data sets in combination with a detailed analysis of the microvascular morphology and modelling provide the comprehensive evidence for a revised understanding of the roles of cerebral arterioles and capillaries in oxygen delivery at rest and during metabolic and haemodynamic perturbations.

Understanding the different roles of arterioles, low- and highbranching-order capillaries in oxygen supply, as well as the dynamic adjustments in the tissue territories that they supply, 
provides a new perspective on cortical microvascular network organization. The energetically demanding cerebral cortex has a large baseline $\mathrm{CBF}$ and low $\mathrm{OEF}$, resulting in high intravascular $\mathrm{PO}_{2}$ that can drive oxygen delivery over increased tissue distances. Taking into account low tissue oxygen diffusivity and high $\mathrm{CMRO}_{2}$, increased cortical tissue distances from vessels in a complex capillary network with very heterogeneous flow and oxygenation (Fig. 6a) may be associated with a greater risk of creating hypoxic tissue islets, the existence of which may be highly undesirable in the brain even for a very brief period of time. Low oxygen delivery from the high-branching-order capillaries in comparison with precapillary arterioles and lowbranching-order capillaries suggests that potentially a significant amount of oxygen diffuses from the tissue back into highbranching-order capillaries and that this may play a role as an oxygen 'mixer', which smoothens the roughness of the tissue oxygenation, reducing the risk of hypoxic tissue islets. This is consistent with previous findings of oxygen exchange between arterioles and capillaries in skeletal muscles ${ }^{25,26}$. In addition, the large capacity of the microvascular network to increase $\mathrm{SO}_{2}$ in high-branching-order capillaries during hyperaemia in combination with dynamic shifting of the tissue supply territories and increase in fractional contribution of highbranching-order capillaries to oxygen delivery during elevated $\mathrm{CMRO}_{2}$ or reduced $\mathrm{CBF}$ ensures that cortical tissue oxygenation is securely maintained in a dynamic brain environment.

Two observed characteristics of the capillary network-(1) a remarkably uniform tissue partitioning by the capillaries independent of the position along the capillary path (Supplementary Fig. 5a) and (2) that high-branching-order capillaries are not contributing much to oxygen delivery at baseline-are raising interesting questions about the signalling that defines the development of the capillary network. It has been shown by modelling that a very basic set of rules such as intense new microvessel sprouting due to tissue hypoxia induced vascular endothelial growth factor (VEGF) production in combination with subsequent pruning based on mechanical stimuli and metabolic state can lead to the generation of a functional vascular network $^{8,27,28}$. Since high-branching-order capillaries are not contributing much to total oxygen release at baseline conditions, the average baseline state tissue oxygenation may not be responsible for their sprouting. It is possible that tissue oxygen fluctuations in a metabolically dynamic brain may generate sufficient vascular endothelial growth factor to shape the morphology of the high-branching-order capillaries. In addition, the mechanical stimuli (for example, vessel wall shear stress and intravascular pressure) may play an important role in their development, as the highly interconnected capillary network achieves rebalancing of flow and asymptotic resistance over distances corresponding to only few capillary segments ${ }^{22}$. Finally, the remarkably uniform tissue partitioning by the capillaries presents the possibility that their development is partially governed by tissue proximity to a source of glucose $\mathrm{e}^{29}$, which has a more uniform distribution along the microvasculature than oxygen, or by removal of the products of metabolism.

The reasons for a large $\mathrm{CBF}$ increase during functional hyperaemia, which causes lower OEF and higher capillary $\mathrm{SO}_{2}$ than in a resting state, has been debated for many years ${ }^{30}$. Some recent evidence ${ }^{93,18}$ suggests that the role of such a high CBF increase is to maintain $\mathrm{PO}_{2}$ at or above the baseline during sustained activation in tissue regions farther away from the microvasculature. The data we present here details two additional conditions in which a large $\mathrm{CBF}$ increase is needed to prevent compromising decreases in tissue $\mathrm{PO}_{2}$. First, capillary $\mathrm{PO}_{2}$ is highly heterogeneous and some capillary segments possibly always have low oxygenation at rest (Fig. 6), which potentially makes tissue in their proximity particularly vulnerable to a sustained increase in oxygen demand. Second, an increase in $\mathrm{CMRO}_{2}$ leads to an enlargement of supplied tissue territories around low-oxygenated capillaries, further exacerbating the problem of maintaining baseline tissue $\mathrm{PO}_{2}$. These statements may need further experimental confirmation since the capillary blood flow regulation during functional activation is multifactorial $^{31,32}$, and potentially not all regulation mechanisms were involved in our steady-state measurements.

The 3D seemingly spatially random structure of the capillary network seems to be important for enabling the close proximity of low- and high-branching-order capillary segments such that their tissue supply territories can interact. In spite of the oxygen mixing in the high-branching-order capillaries due to both flow mixing and oxygen diffusion from tissue back to the capillary blood, capillary $\mathrm{PO}_{2}$ is very heterogeneous (Fig. 6a). This is likely a natural result of the complex capillary network. Moreover, in each data set we found a number of single capillaries as well as groups of connected capillaries (Fig. 1c) with fairly low $\mathrm{PO}_{2}$ $(<15 \mathrm{~mm} \mathrm{Hg})$. This may not mean that tissue $\mathrm{PO}_{2}$ is compromised around these capillary segments, as critical tissue $\mathrm{PO}_{2}$ may only be a few $\mathrm{mm} \mathrm{Hg}^{21,33,34}$. However, it opens the question whether the tissue pockets associated with these capillary domains of lower $\mathrm{PO}_{2}$ may be particularly vulnerable to pathological perturbations such as reduced $\mathrm{CBF}$ in stroke and brain trauma and/or alterations in the capillary morphology in microvasculopathies such as chronic hypertension, diabetes mellitus and Alzheimer's disease ${ }^{12}$. Under pathological stress, these tissue domains may be the initial sites of cortical injury that can further exacerbate the progression of the disease.

We have found that average $\mathrm{SO}_{2}$ in capillaries and postcapillary venules is significantly lower than $\mathrm{SO}_{2}$ in large pial veins-an observation previously made in other tissues? ${ }^{7}$ By using both measurements and modelling we confirmed that this observation can be largely explained by the strong positive correlation between oxygenation and flow along the microvascular paths, such that flow-weighted mean $\mathrm{SO}_{2}$ is significantly higher than the mean $\mathrm{SO}_{2}$ of a population of the microvascular segments. This may be important to consider when assessing oxygen extraction in a number of established brain-imaging modalities that are sensitive to haemoglobin concentration, such as functional magnetic resonance imaging (MRI) and diffuse optical tomography, or emerging technologies such as photoacoustic tomography. Especially, care should be taken when interpreting the data from the parenchyma below the pial surface, where the discrepancy may be the largest. Further measurements and modelling may allow us to gain a detailed understanding of the discrepancy in the mean versus flow-weighted mean $\mathrm{SO}_{2}$ measurements, to develop effective algorithms to correct for it, and to exploit it for development of novel imaging biomarkers. In addition, our detailed quantification of microvascular oxygen distribution at baseline and during elevated CBF, as well as further measurements during functional activation may significantly influence blood-oxygen-level dependent (BOLD)functional MRI signal modelling and interpretation ${ }^{13}$, and the development of novel MRI techniques for detailed quantitative oxygenation measurements in brain ${ }^{14,15}$.

\section{Methods}

The multimodal microscopy imaging set-up. We used a custom-built multimodal microscope set-up in this study (Fig. 1a). The microscope was constructed with a goal to image in vivo cerebral physiology in small rodents and it was designed as a combination of spectral domain OCT and laser scanning TPM. The optimal functioning of each component of the multimodal set-up (for example, OCT and TPM) requires significantly different optical beam properties and scanning protocols. Therefore, to achieve maximal flexibility when designing the experiments and to take full advantage of each imaging modality, 
OCT and TPM modules were designed with separate scanning arms and their probing optical beams were coupled into the same microscope objective by a movable dichroic mirror. The details of the TPM and OCT designs can be found in Sakadzic et al. ${ }^{16}$ and Srinivasan et al. ${ }^{20}$, respectively.

The TPM set-up. The TPM optical beam was scanned in the $x-y$ plane by galvanometer scanners $(6215 \mathrm{H}$, Cambridge Technology Inc.). An electro-optic modulator (ConOptics Inc.; extinction ratio 500) served to gate the output of a Ti:Sapphire oscillator $(840 \mathrm{~nm}, 80 \mathrm{MHz}, 110 \mathrm{fs}$, Mai-Tai, Spectra-Physics). The pulse duration was $\sim 350 \mathrm{fs}$ (assuming a sech ${ }^{2}$ pulse shape), as measured in the sample. The emission was reflected by a dichroic mirror (LP $735 \mathrm{~nm}$; Semrock) and detected with a large collection efficiency $\left(\sim 15 \mathrm{~mm}^{2}\right.$ sr $)$ by a detector array, consisting of four independent photomultiplier tubes. The phosphorescence output was passed through a $680 \pm 30$-nm bandpass filtre and forwarded to a photoncounting photomultiplier tube module (H10770PA-50; Hamamatsu), whose output was acquired by a $50-\mathrm{MHz}$ digital board (NI PCle-6537; National Instruments) and saved for later processing. To determine the phosphorescence lifetime, we fitted the phosphorescence intensity decay with a single-exponential function using the nonlinear least-squares method. The lifetime was converted to $\mathrm{PO}_{2}$ using the calibration plot obtained in independent oxygen titration experiments ${ }^{17}$.

The OCT set-up. A superluminescent diode (model EX8705-2411, Exalos Inc.) with a centre wavelength of $856 \mathrm{~nm}$, bandwidth of $54 \mathrm{~nm}$ and a power of $5 \mathrm{~mW}$ was used as the light source. The axial (depth) resolution was $\sim 6.4 \mu \mathrm{m}$ in air $(4.8 \mu \mathrm{m}$ in tissue) after spectral shaping. The power on the sample was $1.5 \mathrm{~mW}$, enabling a maximum sensitivity of $99 \mathrm{~dB}$. A home-built spectrometer using a 2,048 pixel, 12-bit line scan camera (Aviiva SM2 camera, e2v Semiconductors) enabled an imaging speed of 22,000 axial scans per second and an axial imaging range of $2.5 \mathrm{~mm}$ in air. The transverse resolution was $11 \mu \mathrm{m}$.

Two pairs of the galvanometer mirrors (Cambridge Technology Inc.) were used to independently scan TPM and OCT beams (Fig. 1a). The galvanometer mirrors were relay imaged onto the back focal plane of a microscope objective (Olympus XLUMPLFL20XW/IR, 0.95 numerical apperture, water immersion, $2 \mathrm{~mm}$ working distance). The TMP and OCT optical beams were coupled into the microscope objective by a movable dichroic mirror. A motorized stage controlled the focal position by moving the objective along the vertical axis ( $\mathrm{Z}$ ). Both TPM and OCT systems were controlled by custom-designed software written in LabView (National Instruments).

Animal preparation. For imaging of $\mathrm{PO}_{2}$ in the microvasculature, C57BL/6 mice (male, 25-30 $g, 10-12$ weeks old) were anesthetized by isoflurane $(1-2 \%$ in a mixture of $\mathrm{O}_{2}$ and air) and tracheotomized under constant temperature $\left(37^{\circ} \mathrm{C}\right)$. We then opened a $2.5-\mathrm{mm}$-wide cranial window over the primary SI cortex with the centre of the window in the parietal bone $2 \mathrm{~mm}$ posterior from the bregma and $2 \mathrm{~mm}$ lateral from midline, removed the dura, and sealed the window with a $150-\mu \mathrm{m}$-thick microscope coverslip. We used a catheter in the femoral artery to administer the dyes, to continuously monitor the blood pressure and heart rate, and to sample systemic blood gases $\left(\mathrm{PCO}_{2}\right.$ and $\left.\mathrm{PO}_{2}\right)$ and $\mathrm{pH}$. A capnometer (Micro-CapnoGraph CI240, Columbus Instruments) was used to continuously monitor end-tidal $\mathrm{PCO}_{2}$. A small metal bar was glued to the exposed animal skull and used to both hold the animal head steady under the microscope and adjust the tilt of the head such that the brain surface inside the imaging window is perpendicular to the microscope objective axis.

Animal experiments. Two groups of animals were used to perform the measurements under normocapnic and hypercapnic conditions. During the measurement period, the isoflurane anaesthesia was reduced to $0.7-1.2 \%$. The oxygensensitive dye (PtP-C343) was injected into the vasculature targeting 10-15 $\mu \mathrm{M}$ initial concentration in the blood. During the normocapnia experiments, mice were ventilated with a mixture of air and oxygen and the following physiological parameters were maintained: arterial $\mathrm{PO}_{2}=130 \pm 20 \mathrm{~mm} \mathrm{Hg}$, arterial

$\mathrm{PCO}_{2}=37 \pm 3 \mathrm{~mm} \mathrm{Hg}$ and mean arterial blood pressure $=80-100 \mathrm{~mm} \mathrm{Hg}$. During the hypercapnia experiments, we added $\sim 5 \%$ of $\mathrm{CO}_{2}$ to the mixture of air and oxygen to achieve arterial $\mathrm{PCO}_{2}=48 \pm 3 \mathrm{~mm} \mathrm{Hg}$. When physiological parameters fell outside the specified ranges during the experiment, we rejected the whole experiment.

The experimental protocol for each animal consisted of $\sim 10$-min-long OCT imaging of CBF followed by the $\sim 30$-min-long TPM imaging of intravascular $\mathrm{PO}_{2}$ and an additional OCT imaging of CBF. The experiments were rejected if the blood flow was not maintained steady as indicated by the CBF change for $>10 \%$ before and after $\mathrm{PO}_{2}$ measurement. At the end of each experiment, we labelled the blood plasma with the fluorescein isothiocyanate (FITC) conjugated with dextran and used TPM to obtain the high-resolution structural image of the microvasculature inside a $725 \times 725-\mu \mathrm{m}$-wide and $\sim 650-\mu \mathrm{m}$-deep region of interest. We also obtained a larger image of the surface microvasculature $(2 \times 2 \mathrm{~mm})$, which was later used for easier tracing of the pial arterioles and venules. We performed normocapnia and hypercapnia experiments on different animal groups due to the challenges of maintaining very stable anesthetized mouse physiology for a long time, which will be required to perform both measurements in the same animal. However, the measurement results within each animal group (that is, normocapnic and hypercapnic animals) were highly reproducible with well-separated means between the groups, which abolished the need for the within-animal comparisons.

The details of the OCT-scanning protocol for the imaging of CBF can be found in Srinivasan et al. ${ }^{20}$ For the TPM imaging of the intravascular $\mathrm{PO}_{2}$, we excited phosphorescence by trains of femtosecond pulses from a Ti:sapphire oscillator, gated by an electro-optic modulator, and acquired decays by averaging multiple excitation cycles. Each cycle consisted of a 10- $\mu$ s excitation gate, followed by a 290- $\mu$ s collection period. At each imaging plane, we performed detection in two steps. First, we raster-scanned the excitation beam over the field of view, rendering two-dimensional survey maps of the integrated phosphorescence emission intensity $(250 \times 250$ pixels, acquired in $\sim 19 \mathrm{~s})$, which revealed the position of the microvasculature in the plane. We then averaged $\sim 2,000$ phosphorescence decays in selected locations in the vasculature for accurate $\mathrm{PO}_{2}$ determination, resulting in 0.6 s per single-point $\mathrm{PO}_{2}$ measurement. $\mathrm{PO}_{2}$ data were collected during $30-40$ min at $400-500$ locations in $30-50 \mu \mathrm{m}$ separated planes up to the $450-\mu \mathrm{m}$ depth. The high similarity of the TPM microvascular structural images of blood plasma labelled by FITC and based on phosphorescence survey scans allowed a simple co-registration of the $\mathrm{PO}_{2}$ measurements with the 3D microvascular stack.

All experimental procedures were approved by the Massachusetts General Hospital Subcommittee on Research Animal Care.

Microvascular segmentation and data co-registration. The segmentation of the microvasculature from the 3D FITC stacks obtained by TPM was performed using a combination of the custom-written software for manual and semi-automatic graphing ${ }^{35}$ and the publicly available VIDA software ${ }^{36}$. After obtaining the mathematical graph-representation of the microvasculature, all microvascular segments (microvascular sections between two consecutive bifurcation points) were labelled as arterioles, venules or capillaries (Fig. la,c). All arterioles and venules were labelled manually by following them from the pial surface into the cortical depth. After labelling the arterioles and venules, all remaining microvessels were labelled as capillaries and then individually visualized and inspected. The identification of the pial arterioles and venules was done based on their morphology and $\mathrm{PO}_{2}$ measurements, and confirmed by tracing them in the largerfield-of-view TPM images $(\sim 2 \times 2 \mathrm{~mm})$ of the surface vasculature. Pial arterioles are straighter than pial veins, and they more gradually branch into smaller vessels. They can be easily distinguished from pial veins, which are curvier, thicker and frequently sprouting smaller vessels of random calibres. Capillaries were typically identified starting one or two segments away from the diving arterioles and surfacing venules based on their morphology (that is, smaller diameters and higher tortuosity).

We used the Floyd-Warshall algorithm ${ }^{37}$ to calculate the shortest distance by either branching order or pathlength along the vascular segments between any two vertices (end points of the vascular segments) in a graph of segmented microvasculature. In addition, we computed the predecessor matrix that provides for each pair of graph vertices $(m, n)$ an index of the vertice $(k)$, which precedes vertice $n$ along the shortest path between $m$ and $n$. This information was used to simply determine all segments along the shortest path between each pair of vertices. We subsequently computed for each vascular segment its minimal branching order and minimal pathlength to the manually identified pial arterioles and venules. We also computed for each capillary segment its minimal branching order and minimal pathlength to the closest precapillary arteriole and postcapillary venule.

The co-registration of TPM and OCT data was performed using the customwritten software in Matlab (MathWorks Inc.). We manually identified numerous pairs of points in both the $3 \mathrm{D}$ velocity projection map obtained by OCT and microvascular stack obtained by TPM, with each pair of points representing the same location in the microvasculature. On the basis of the selected pairs of points, the OCT velocity projection map and computed absolute flow in selected vascular segments were spatially transformed onto the TPM microvascular stack (Supplementary Fig. 1).

Numerical simulations. We utilized a VAN model developed by Fang et al..$^{35}$ and Gagnon et $_{\text {al. }}{ }^{38}$ to compute the resting-state flow and resting-state oxygenation in both microvasculature and tissue in four microvascular stacks obtained in our normocapnia and hypercapnia experiments. We removed the capillary segments cut by the limits of the vascular stacks to obtain a closed graph between the pial arteries and veins and to reduce the number of places where the boundary conditions must be set ${ }^{39}$. We fixed CBF in computations to obtain a perfusion of $\sim 1 \mathrm{mlg}^{-1} \mathrm{~min}^{-1}$ in our volumes-a value recently reported in mouse cortices under the same anaesthesia ${ }^{40,41}$. We also fixed $\mathrm{PO}_{2}$ in the pial arterioles to $\sim 100 \mathrm{~mm} \mathrm{Hg}$ and tissue $\mathrm{CMRO}_{2}$ to $\sim 2.3 \mu \mathrm{molO}_{2} \mathrm{~g}^{-1} \mathrm{~min}^{-1}$ to obtain a reasonable match between computed and measured intravascular oxygen distributions. The oxygen advection was run with constant inputs until a steady state was achieved (typically after $15 \mathrm{~s}$ in model time). To avoid the artefacts due to the vascular stack boundaries, only the computed variables from the central region of the microvascular stacks (up to $100 \mu \mathrm{m}$ inside from the lateral sides and from the upper $450 \mu \mathrm{m}$ of each stack) were used in statistical analyses.

$\mathbf{P O}_{\mathbf{2}}$ fitting and calculation of $\mathbf{S O}_{\mathbf{2}}$. In all measurements, we compared the phosphorescence survey scans taken immediately before and after $\mathrm{PO}_{2}$ point 
measurements in each plane and inspected collected phosphorescence decays for signs of sudden intensity or decay-slope change during acquisition, which could indicate motion artefact during the measurement. To determine the phosphorescence lifetime, we fitted the phosphorescence intensity decay with a single-exponential function using the nonlinear least-squares method. The lifetime was converted to $\mathrm{PO}_{2}$ using the calibration plot obtained in independent oxygen titration experiments ${ }^{17}$. The s.d. of the $\mathrm{PO}_{2}$ measurement generally increased with $\mathrm{PO}_{2}$ up to $4 \mathrm{~mm} \mathrm{Hg}$ at high $\mathrm{PO}_{2}$ values. We rejected $\mathrm{PO}_{2}$ measurements with a relative s.e. $>15 \% . \mathrm{PO}_{2}$ inside the capillaries was estimated in the blood plasma adjacent to the red blood cells, following the procedure outlined in Parpaleix et al..$^{42}$ We subsequently computed the $\mathrm{SO}_{2}$ based on the Hill equation, with Hill coefficients specific for C57BL/6 mice $\left(h=2.59 \text { and } P_{50}=40.2 \mathrm{~mm} \mathrm{Hg}\right)^{43}$.

Statistical analysis of the capillary branching orders and pathlengths. On the basis of the mathematical graph-representation of the microvasculature in six microvascular stacks, we first identified vascular segment vertices that separate arteriolar and capillary segments. We subsequently computed for each capillary segment its minimal branching order and minimal distance along the capillary path to the closest precapillary arteriole and postcapillary venule, taking into account only microvascular paths without arteriolar and venular segments. In addition, to minimize the effect of incomplete capillary paths due to a finite size of microvascular stacks, we considered only the capillary segments $100 \mu \mathrm{m}$ away from the lateral boundaries of the field of view and up to $450 \mu \mathrm{m}$ deep from the cortical surface. The findings about capillary branching orders and pathlengths based only on microvascular morphology were further confirmed by following the capillary paths along the downstream blood flow computed by VAN modelling in four realistic microvascular networks.

\section{Estimation of the intra-arteriolar radial $\mathrm{PO}_{2}$ profiles. Intra-arteriolar mea-} surements were performed in three mice (normoxic normocapnia), in 4 diving arterioles, $50-150 \mu \mathrm{m}$ below the cortical surface. The $\mathrm{PO}_{2}$ inside the vessel crosssection was measured in a dense rectangular grid (Fig. 2b). Typically $>200 \mathrm{PO}_{2}$ measurements were obtained. Starting from the centre of the vessel, measurements from 1- $\mu \mathrm{m}$-thick rings were grouped and used to calculate the mean $\mathrm{PO}_{2}$ and s.e. at different radial distances from the vessel centre (Fig. 2c). Starting from the centre of each measured arteriole, measurements from 3 - $\mu \mathrm{m}$-thick rings were grouped and the Student's $t$-test was performed to find a minimal radius with the mean $\mathrm{PO}_{2}$ significantly lower $(\alpha=0.05)$ than $\mathrm{PO}_{2}$ at the vessel centre.

Construction of composite images. We colour-coded the vascular segments in composite images (Fig. 1) by assigning a mean measured $\mathrm{PO}_{2}$ value in that segment to the whole vessel segment between two branching points. In addition, to allow more complete visualization of the oxygenation in the vascular tree when creating the composite images, for segments that did not have a measurement but joined at both ends with segments that had measurements within $100 \mu \mathrm{m}$, we assigned the average $\mathrm{PO}_{2}$ value of the connecting segments.

\section{References}

1. Attwell, D. \& Laughlin, S. B. An energy budget for signaling in the grey matter of the brain. J. Cereb. Blood Flow Metab. 21, 1133-1145 (2001).

2. Duling, B., Kuschinsky, W. \& Wahl, M. Measurements of the perivascular $\mathrm{PO}_{2}$ in the vicinity of the pial vessels. Pflugers Arch. 383, 29-34 (1979).

3. Vovenko, E. Distribution of oxygen tension on the surface of arterioles, capillaries and venules of brain cortex and in tissue in normoxia: an experimental study on rats. Pflugers Arch. 437, 617-623 (1999).

4. Vazquez, A. L., Masamoto, K., Fukuda, M. \& Kim, S.-G. Cerebral oxygen delivery and consumption during evoked neural activity. Front. Neuroenergetics 2, $11(2010)$

5. Duling, B. R. \& Berne, R. M. Longitudinal gradients in periarteriolar oxygen tension a possible mechanism for the participation of oxygen in local regulation of blood flow. Circ. Res. 27, 669-678 (1970).

6. Krogh, A. The supply of oxygen to the tissues and the regulation of the capillary circulation. J. Physiol. 52, 457-474 (1919).

7. Tsai, A. G., Johnson, P. C. \& Intaglietta, M. Oxygen gradients in the microcirculation. Physiol. Rev. 83, 933-963 (2003).

8. Secomb, T. W., Alberding, J. P., Hsu, R., Dewhirst, M. W. \& Pries, A. R. Angiogenesis: an adaptive dynamic biological patterning problem. PLoS Comput. Biol. 9, e1002983 (2013).

9. Pittman, R. Oxygen transport and exchange in the microcirculation. Microcirculation 12, 59-70 (2005).

10. Popel, A. Theory of oxygen-transport to tissue. Crit. Rev. Biomed. Eng. 17, 257-321 (1989).

11. Goldman, D. Theoretical models of microvascular oxygen transport to tissue. Microcirculation 15, 795-811 (2008)

12. Zlokovic, B. V. Neurovascular pathways to neurodegeneration in Alzheimer's disease and other disorders. Nat. Rev. Neurosci. 12, 723-738 (2011).
13. Buxton, R. B. Interpreting oxygenation-based neuroimaging signals: the importance and the challenge of understanding brain oxygen metabolism. Front. Neuroenergetics 2, 8 (2010).

14. Bolar, D. S., Rosen, B. R., Sorensen, A. G. \& Adalsteinsson, E. QUantitative Imaging of eXtraction of Oxygen and TIssue consumption (QUIXOTIC) using venular-targeted velocity-selective spin labeling. Magn. Reson. Med. 66, 1550-1562 (2011).

15. Ma, D. et al. Magnetic resonance fingerprinting. Nature 495, 187-192 (2013).

16. Sakadzic, S. et al. Two-photon high-resolution measurement of partial pressure of oxygen in cerebral vasculature and tissue. Nat. Methods 7, 755-U125 (2010).

17. Finikova, O. S. et al. Oxygen microscopy by two-photon-excited phosphorescence. Chemphyschem 9, 1673-1679 (2008).

18. Devor, A. et al. 'Overshoot' of $\mathrm{O} 2$ is required to maintain baseline tissue oxygenation at locations distal to blood vessels. J. Neurosci. 31, 13676-13681 (2011).

19. Lecoq, J. et al. Simultaneous two-photon imaging of oxygen and blood flow in deep cerebral vessels. Nat. Med. 17, 893-U262 (2011).

20. Srinivasan, V. J. et al. Quantitative cerebral blood flow with optical coherence tomography. Opt. Express 18, 2477-2494 (2010).

21. Kasischke, K. A. et al. Two-photon NADH imaging exposes boundaries of oxygen diffusion in cortical vascular supply regions. J. Cereb. Blood Flow Metab. 31, 68-81 (2011)

22. Blinder, P. et al. The cortical angiome: an interconnected vascular network with noncolumnar patterns of blood flow. Nat. Neurosci. 16, 889-897 (2013).

23. Masamoto, K., Kurachi, T., Takizawa, N., Kobayashi, H. \& Tanishita, K. Successive depth variations in microvascular distribution of rat somatosensory cortex. Brain Res. 995, 66-75 (2004).

24. Hall, J. E. Guyton and Hall Textbook of Medical Physiology (Saunders, 2010).

25. Secomb, T. W. \& Hsu, R. Simulation of O2 transport in skeletal muscle: diffusive exchange between arterioles and capillaries. Am. J. Physiol. 267, H1214-H1221 (1994).

26. Ellsworth, M. \& Pittman, R. Arterioles supply oxygen to capillaries by diffusion as well as by convection. Am. J. Physiol. 258, H1240-H1243 (1990).

27. Munn, L. L., Kunert, C. \& Tyrrell, J. A. in Mathematical Methods and Models in Biomedicine (eds Ledzewicz, U., Schättler, H., Friedman, A. \& Kashdan, E.) 117-148 (Springer, 2012).

28. Pries, A. R. \& Secomb, T. W. in Comprehensive Physiology 3-36 (John Wiley \& Sons Inc., 2011).

29. Paulson, O. B., Hasselbalch, S. G., Rostrup, E., Knudsen, G. M. \& Pelligrino, D Cerebral blood flow response to functional activation. J. Cereb. Blood Flow Metab. 30, 2-14 (2010).

30. Fox, P. \& Raichle, M. Focal physiological uncoupling of cerebral blood-flow and oxidative-metabolism during somatosensory stimulation in human-subjects. Proc. Natl Acad. Sci. USA 83, 1140-1144 (1986).

31. Hall, C. N. et al. Capillary pericytes regulate cerebral blood flow in health and disease. Nature 508, 55-60 (2014).

32. Jespersen, S. N. \& Ostergaard, L. The roles of cerebral blood flow, capillary transit time heterogeneity, and oxygen tension in brain oxygenation and metabolism. J. Cereb. Blood Flow Metab. 32, 264-277 (2012).

33. Chance, B. Cellular oxygen requirements. Fed. Proc. 16, 671-680 (1957).

34. Wilson, D. F., Harrison, D. K. \& Vinogradov, S. A. Oxygen, pH, and mitochondrial oxidative phosphorylation. J. Appl. Physiol. 113, 1838-1845 (2012).

35. Fang, Q. et al. Oxygen advection and diffusion in a three- dimensional vascular anatomical network. Opt. Express 16, 17530-17541 (2008).

36. Tsai, P. S. et al. Correlations of neuronal and microvascular densities in murine cortex revealed by direct counting and colocalization of nuclei and vessels. J. Neurosci. 29, 14553-14570 (2009).

37. Floyd, R. Algorithm-97-shortest path. Commun. Acm 5, 345-345 (1962).

38. Gagnon, L. et al. Underpinning the microvascular origin of BOLD-fMRI with two-photon microscopy, in Biomedical Optics 2014, BT4A.1 (Optical Society of America, 2014).

39. Lorthois, S., Cassot, F. \& Lauwers, F. Simulation study of brain blood flow regulation by intra-cortical arterioles in an anatomically accurate large human vascular network: Part I: methodology and baseline flow. Neuroimage 54, 1031-1042 (2011).

40. Wehrl, H. F. et al. Simultaneous assessment of Perfusion with [15O] water PET and arterial spin labeling MR using a hybrid PET/MR device. Proc. Intl Soc. Mag. Reson. Med. 18, 715 (2010).

41. Zheng, B., Lee, P. T. H. \& Golay, X. High-sensitivity cerebral perfusion mapping in mice by kbGRASE-FAIR at 9.4 T. NMR Biomed. 23, 1061-1070 (2010).

42. Parpaleix, A., Houssen, Y. G. \& Charpak, S. Imaging local neuronal activity by monitoring $\mathrm{PO}_{2}$ transients in capillaries. Nat. Med. 19, 241-246 (2013).

43. Uchida, K., Reilly, M. P. \& Asakura, T. Molecular stability and function of mouse hemoglobins. Zoolog. Sci. 15, 703-706 (1998). 


\section{Acknowledgements}

We would like to thank Sergei A. Vinogradov and Emmanouil Rousakis for providing the oxygen-sensitive dye and for their help with the $\mathrm{PO}_{2}$ imaging. We would also like to thank support from the American Heart Association (grant SDG7600037) and from the National Institute of Health (grants EB000790, NS055104, NS057476 and K99AG042026).

\section{Author contributions}

S.S. and D.A.B. designed the study with input from A.D., C.A., E.T.M., M.A. Yaseen, V.J.S., E.H.L., A.M.D. and K.E.-H. S.S. analysed the data and wrote the manuscript with help from D.A.B. and all other authors. S.S., E.T.M. and K.E.-H. performed the experiments. S.S., M.A. Yaseen and V.J.S. built the microscope. L.G., S.S. and D.A.B. performed the numerical simulations. J.J.M., S.S., L.G., M.A. Yucel, J.L. and F.L. carried out segmentation of the microvascular stacks.

\section{Additional information}

Supplementary Information accompanies this paper at http://www.nature.com/ naturecommunications

Competing financial interests: The authors declare no competing financial interests.

Reprints and permission information is available online at http://npg.nature.com/ reprintsandpermissions/

How to cite this article: Sakadžić, S. et al. Large arteriolar component of oxygen delivery implies a safe margin of oxygen supply to cerebral tissue. Nat. Commun. 5:5734 doi: 10.1038/ncomms6734 (2014). 